KYUNGPOOK Math. J. 00(0000), 000-000

\title{
On a Class of Meromorphic Functions defined by Certain Lin- ear Operators
}

S. Sivaprasad Kumar*

Department of Applied Mathematics, Delhi College of Engineering, Delhi - 110 042,

India

e-mail : spkumar@dce.ac.in

H. C. TANEJA

Department of Applied Mathematics, Delhi College of Engineering, Delhi - 110 042,

India

e-mail : hctaneja@dce.ac.in

Abstract. In the present investigation, we introduce new classes of $p$-valent meromorphic functions defined by Liu-Srivastava linear operator and the multiplier transform and study their properties by using certain first order differential subordination and superordination.

\section{Introduction}

Let $\mathscr{H}$ be the class of functions analytic in $\Delta:=\{z \in \mathbb{C}:|z|<1\}$ and $\mathscr{H}(a, n)$ be the subclass of $\mathscr{H}$ consisting of functions of the form $f(z)=$ $a+a_{n} z^{n}+a_{n+1} z^{n+1}+\cdots$ and set $\mathscr{H}_{1}:=\mathscr{H}(1,1)$. Let $\Sigma_{p}$ denote the class of all analytic functions of the form

$$
f(z)=\frac{1}{z^{p}}+\sum_{k=1-p}^{\infty} a_{k} z^{k} \quad\left(z \in \Delta^{*}:=\{z \in \mathbb{C}: 0<|z|<1\} ; p \in \mathbb{N}\right)
$$

and set $\Sigma:=\Sigma_{1}$

For two functions $f(z)$ given by $(1.1)$ and $g(z)=\frac{1}{z^{p}}+\sum_{k=1-p}^{\infty} b_{k} z^{k}$, the Hadamard product (or convolution) of $f$ and $g$ is defined by

$$
(f * g)(z):=\frac{1}{z^{p}}+\sum_{k=1-p}^{\infty} a_{k} b_{k} z^{k}=:(g * f)(z) .
$$

For $\alpha_{j} \in \mathbb{C} \quad(j=1,2, \cdots, l)$ and $\beta_{j} \in \mathbb{C} \backslash\{0,-1,-2, \cdots\}(j=1,2, \cdots m)$, the generalized hypergeometric function ${ }_{l} F_{m}\left(\alpha_{1}, \cdots, \alpha_{l} ; \beta_{1}, \cdots, \beta_{m} ; z\right)$ is defined by the

\footnotetext{
* Corresponding author.
}

Received August 29, 2008; accepted December 11, 2008.

2000 Mathematics Subject Classification: Primary 30C45, Secondary 30C80.

Key words and phrases: hypergeometric functions, subordination, linear operator, multiplier transform, convolution, Differential superordinations, subordinant, dominant. 
infinite series

$$
\begin{aligned}
{ }_{l} F_{m}\left(\alpha_{1}, \cdots, \alpha_{l} ; \beta_{1}, \cdots, \beta_{m} ; z\right) & :=\sum_{n=0}^{\infty} \frac{\left(\alpha_{1}\right)_{n} \cdots\left(\alpha_{l}\right)_{n}}{\left(\beta_{1}\right)_{n} \cdots\left(\beta_{m}\right)_{n}} \frac{z^{n}}{n !} \\
\left(l \leq m+1 ; l, m \in \mathbb{N}_{0}\right. & :=\{0,1,2, \cdots\})
\end{aligned}
$$

where $(a)_{n}$ is the Pochhammer symbol defined by

$$
(a)_{n}:=\frac{\Gamma(a+n)}{\Gamma(a)}= \begin{cases}1, & (n=0) ; \\ a(a+1)(a+2) \ldots(a+n-1), & (n \in \mathbb{N}:=\{1,2,3 \cdots\}) .\end{cases}
$$

Corresponding to the function

$$
h_{p}\left(\alpha_{1}, \cdots, \alpha_{l} ; \beta_{1}, \cdots, \beta_{m} ; z\right):=z^{-p}{ }_{l} F_{m}\left(\alpha_{1}, \cdots, \alpha_{l} ; \beta_{1}, \cdots, \beta_{m} ; z\right),
$$

the Liu-Srivastava operator [7] $\mathcal{H}_{p}^{(l, m)}\left(\alpha_{1}, \cdots, \alpha_{l} ; \beta_{1}, \cdots, \beta_{m}\right): \Sigma_{p} \mapsto \Sigma_{p}$ is defined by the Hadamard product

$$
\begin{aligned}
\mathcal{H}_{p}^{(l, m)}\left(\alpha_{1}, \cdots, \alpha_{l} ; \beta_{1}, \cdots, \beta_{m}\right) f(z) & :=h_{p}\left(\alpha_{1}, \cdots, \alpha_{l} ; \beta_{1}, \cdots, \beta_{m} ; z\right) * f(z) \\
& =\frac{1}{z^{p}}+\sum_{n=1-p}^{\infty} \frac{\left(\alpha_{1}\right)_{n+p} \cdots\left(\alpha_{l}\right)_{n+p}}{\left(\beta_{1}\right)_{n+p} \cdots\left(\beta_{m}\right)_{n+p}} \frac{a_{n} z^{n}}{(n+p) !} .
\end{aligned}
$$

To make the notation simple, we write

$$
\mathcal{H}_{p}^{l, m}\left[\alpha_{1}\right] f(z):=\mathcal{H}_{p}^{(l, m)}\left(\alpha_{1}, \cdots, \alpha_{l} ; \beta_{1}, \cdots, \beta_{m}\right) f(z) .
$$

Special cases of the Liu-Srivastava linear operator includes the meromorphic analogue of the Carlson-Shaffer linear operator

$$
\mathcal{L}_{p}(a, c):=\mathcal{H}_{p}^{(2,1)}(a, 1 ; c)
$$

considered by Liu [6] and the operator

$$
\mathcal{D}^{n+p-1}:=\mathcal{L}_{p}(n+p, 1)
$$

investigated by Yang [14]. When $p=1$, the operator was first introduced by Ganigi and Uralegaddi [5] and then generalized by Yang [13] to an operator analogous to the Ruscheweyh derivative operator, and the operator $\mathcal{J}_{c, p}=\mathcal{L}_{p}(c, c+1)$ was studied, for example, by Uralegaddi and Somanatha [12]. Note that

$$
\mathcal{J}_{c, p} f=\frac{c}{z^{c+p}} \int_{0}^{z} t^{c+p-1} f(t) d t \quad(c>0) .
$$

Motivated by the operator studied by Aouf and Hossen [1] (see also [4], [6], [10]), we define the operator $\mathcal{I}_{p}(n, \lambda)$ on $\Sigma_{p}$ by the following infinite series

$$
\mathcal{I}_{p}(n, \lambda) f(z):=\frac{1}{z^{p}}+\sum_{k=1-p}^{\infty}\left(\frac{k+\lambda}{\lambda-p}\right)^{n} a_{k} z^{k} \quad(\lambda>p, n \in \mathbb{Z}) .
$$


A function $f(z) \in \Sigma_{p}$ is said to be in the class $\Omega_{p}^{l, m}\left(\alpha_{1} ; A, B\right)$ if it satisfies the following subordination:

$$
\begin{gathered}
\frac{\left(\mathcal{H}_{p}^{l, m}\left[\alpha_{1}+1\right] f(z)\right)^{\prime}}{\left(\mathcal{H}_{p}^{l, m}\left[\alpha_{1}\right] f(z)\right)^{\prime}} \prec 1-\frac{p(A-B) z}{\alpha_{1}(1+B z)} \\
\left(z \in \Delta ; \quad \alpha_{1} \in \mathbb{C} ;-1<B<A \leq 1 ; p, l, m \in \mathbb{N}\right)
\end{gathered}
$$

This class $\Omega_{p}^{l, m}\left(\alpha_{1} ; A, B\right)$ was introduced by Liu and Srivastava [7] and they have proved the following:

Theorem 1.1([7, Theorem 1, p.23]). Let $\alpha_{1}$ be real number. If

$$
\alpha_{1} \geq \frac{p(A-B)}{1+B} \quad(-1<B<A \leq 1 ; p \in \mathbb{N}),
$$

then

$$
\Omega_{p}^{l, m}\left(\alpha_{1}+1 ; A, B\right) \subset \Omega_{p}^{l, m}\left(\alpha_{1} ; A, B\right) .
$$

Theorem 1.2([7, Theorem 2, p.25]). Let $\lambda$ be a complex number such that

$$
\Re(\lambda)>\frac{p(A-B)}{1+B} \quad(-1<B<A \leq 1 ; p \in \mathbb{N}) .
$$

If $f \in \Omega_{p}^{l, m}\left(\alpha_{1} ; A, B\right)$, then the function

$$
F(z)=\frac{\lambda}{z^{\lambda+p}} \int_{0}^{z} t^{\lambda+p-1} f(t) d t
$$

also belongs to the class $\Omega_{p}^{l, m}\left(\alpha_{1} ; A, B\right)$.

For two analytic functions $f$ and $F$, we say that $F$ is superordinate to $f$ if $f$ is subordinate to $F$. Recently Miller and Mocanu [9] considered certain second order differential superordinations. Using the results of Miller and Mocanu [9], Bulboaca [3] has considered certain classes of first order differential superordinations and Bulboaca [2] considered certain superordination-preserving integral operators.

In recent years, many results of various interesting subclasses of the class $\Sigma_{p}$ of meromorphically $p$-valent functions were investigated extensively by (among others) Aouf et al. [1], Liu and Srivasava [7], Ravichandran et al. [11], Uralegaddi and Somanatha [12] and Yang [14]. In this paper, we generalize the above-stated classes of Liu and Srivasava [7] to a more general classes of meromorphic $p$-valent functions which we define below using differential subordination and superordination.

Definition 1.1. A function $f(z) \in \Sigma_{p}$ is said to be in $\Omega_{p}\left(\alpha_{1}, \cdots, \alpha_{l} ; \beta_{1}, \cdots, \beta_{m} ; \varphi\right)$ if it satisfies the following subordination:

$$
\frac{\left(\mathcal{H}_{p}^{l, m}\left[\alpha_{1}+1\right] f(z)\right)^{\prime}}{\left(\mathcal{H}_{p}^{l, m}\left[\alpha_{1}\right] f(z)\right)^{\prime}} \prec \varphi(z) \quad(z \in \Delta),
$$


and is said to be in $\widetilde{\Omega}_{p}\left(\alpha_{1}, \cdots, \alpha_{l} ; \beta_{1}, \cdots, \beta_{m} ; \varphi\right)$ if $f$ satisfies the following superordination:

$$
\varphi(z) \prec \frac{\left(\mathcal{H}_{p}^{l, m}\left[\alpha_{1}+1\right] f(z)\right)^{\prime}}{\left(\mathcal{H}_{p}^{l, m}\left[\alpha_{1}\right] f(z)\right)^{\prime}} \quad(z \in \Delta),
$$

where $\varphi(z)$ is analytic in $\Delta$ and $\varphi(0)=1$.

To make the notation simple, we write

$$
\Omega_{p}\left(\alpha_{1} ; \varphi\right):=\Omega_{p}\left(\alpha_{1}, \cdots, \alpha_{l} ; \beta_{1}, \cdots, \beta_{m} ; \varphi\right)
$$

and

$$
\widetilde{\Omega}_{p}\left(\alpha_{1} ; \varphi\right):=\widetilde{\Omega}_{p}\left(\alpha_{1}, \cdots, \alpha_{l} ; \beta_{1}, \cdots, \beta_{m} ; \varphi\right) .
$$

Also we define the class $\Omega_{p}\left(\alpha_{1} ; \varphi_{1}, \varphi_{2}\right)$ by the following:

$$
\Omega_{p}\left(\alpha_{1} ; \varphi_{1}, \varphi_{2}\right):=\widetilde{\Omega}_{p}\left(\alpha_{1} ; \varphi_{1}\right) \cap \Omega_{p}\left(\alpha_{1} ; \varphi_{2}\right) .
$$

For

$$
\varphi(z)=1-\frac{p(A-B) z}{\alpha_{1}(1+B z)} \quad\left(z \in \Delta ; \quad \alpha_{1} \in \mathbb{C} ;-1<B<A \leq 1 ; p \in \mathbb{N}\right),
$$

the class $\Omega_{p}\left(\alpha_{1} ; \varphi\right)$ reduces to the class $\Omega_{p}^{l, m}\left(\alpha_{1} ; A, B\right)$, introduced and studied by Liu and Srivastava [7].

Definition 1.2. A function $f(z) \in \Sigma_{p}$ is said to be in the class $\mathcal{M}_{p}(n, \lambda ; \varphi)$ if it satisfies the following subordination:

$$
\frac{\left(\mathcal{I}_{p}(n+1, \lambda) f(z)\right)^{\prime}}{\left(\mathcal{I}_{p}(n, \lambda) f(z)\right)^{\prime}} \prec \varphi(z) \quad\left(f(z) \in \Sigma_{p}\right),
$$

and is said to be in $\widetilde{\mathcal{M}}_{p}(n, \lambda ; \varphi)$ if $f$ satisfies the following superordination:

$$
\varphi(z) \prec \frac{\left(\mathcal{I}_{p}(n+1, \lambda) f(z)\right)^{\prime}}{\left(\mathcal{I}_{p}(n, \lambda) f(z)\right)^{\prime}} \quad\left(f(z) \in \Sigma_{p}\right),
$$

where $\varphi(z)$ is analytic in $\Delta$ and $\varphi(0)=1$. Also we define the class $\mathcal{M}_{p}\left(n, \lambda ; \varphi_{1}, \varphi_{2}\right)$ by the following:

$$
\mathcal{M}_{p}\left(n, \lambda ; \varphi_{1}, \varphi_{2}\right):=\widetilde{\mathcal{M}}_{p}\left(n, \lambda ; \varphi_{1}\right) \cap \mathcal{M}_{p}\left(n, \lambda ; \varphi_{2}\right) .
$$

\section{Preliminaries}

In order to prove our main results we will need to use the next definition and lemmas. 
Definition 2.1 ([9, Definition 2, p.817]). Denote by $\mathcal{Q}$, the set of all functions $f(z)$ that are analytic and injective on $\bar{\Delta}-E(f)$, where

$$
E(f)=\left\{\zeta \in \partial \Delta: \lim _{z \rightarrow \zeta} f(z)=\infty\right\},
$$

and are such that $f^{\prime}(\zeta) \neq 0$ for $\zeta \in \partial \Delta-E(f)$.

Lemma 2.1([8]). Let $q(z)$ be univalent in the unit disk $\Delta$ and $\vartheta$ and $\varphi$ be analytic in a domain $\mathbb{D}$ containing $q(\Delta)$ with $\varphi(w) \neq 0$ when $w \in q(\Delta)$. Set $Q(z):=z q^{\prime}(z) \varphi(q(z)), h(z):=\vartheta(q(z))+Q(z)$. Suppose that either (i) $h(z)$ is convex, or (ii) $Q(z)$ is starlike univalent in $\Delta$. In addition, assume that

$$
\Re \frac{z h^{\prime}(z)}{Q(z)}>0 \quad(z \in \Delta) .
$$

If $p(z)$ is analytic in $\Delta$, with $p(0)=q(0), p(\Delta) \subset \mathbb{D}$ and

$$
\vartheta(p(z))+z p^{\prime}(z) \varphi(p(z)) \prec \vartheta(q(z))+z q^{\prime}(z) \varphi(q(z))=h(z),
$$

then $p(z) \prec q(z)$ and $q(z)$ is the best dominant.

Lemma 2.2([3]). Let $q(z)$ be univalent in the unit disk $\Delta$ and $\vartheta$ and $\varphi$ be analytic in a domain $\mathbb{D}$ containing $q(\Delta)$. Suppose that

(1) $\Re\left[\vartheta^{\prime}(q(z)) / \varphi(q(z))\right]>0$ for $z \in \Delta$,

(2) $Q(z):=z q^{\prime}(z) \varphi(q(z))$ is starlike univalent in $\Delta$.

If $p(z) \in \mathscr{H}[q(0), 1] \cap \mathcal{Q}$, with $p(\Delta) \subset \mathbb{D}$, and $\vartheta(p(z))+z p^{\prime}(z) \varphi(p(z))$ is univalent in $\Delta$, then

$$
\vartheta(q(z))+z q^{\prime}(z) \varphi(q(z)) \prec \vartheta(p(z))+z p^{\prime}(z) \varphi(p(z)),
$$

implies $q(z) \prec p(z)$ and $q(z)$ is the best subordinant.

3. The classes $\Omega_{p}\left(\alpha_{1} ; \varphi\right)$ and $\widetilde{\Omega}_{p}\left(\alpha_{1} ; \varphi\right)$

By making use of Lemma 2.1, we first prove the following result:

Theorem 3.1. Let $\psi(z)$ be univalent in $\Delta, \psi(0)=1$ and $\psi(z) \neq 0$. Assume that $z \psi^{\prime} / \psi$ is starlike in $\Delta$ and $\Re\left\{\alpha_{1} \psi(z)\right\}>0$. Let $\chi(z)$ be defined by

$$
\chi(z):=\frac{1}{\alpha_{1}+1}\left[\alpha_{1} \psi(z)+1+\frac{z \psi^{\prime}(z)}{\psi(z)}\right] .
$$

If $f(z) \in \Omega_{p}\left(\alpha_{1}+1 ; \chi\right)$, then $f(z) \in \Omega_{p}\left(\alpha_{1} ; \psi\right)$. If $f(z) \in \widetilde{\Omega}_{p}\left(\alpha_{1}+1 ; \chi\right)$,

$$
0 \neq \frac{\left(\mathcal{H}_{p}^{l, m}\left[\alpha_{1}+1\right] f(z)\right)^{\prime}}{\left(\mathcal{H}_{p}^{l, m}\left[\alpha_{1}\right] f(z)\right)^{\prime}} \in \mathscr{H}_{1} \cap \mathcal{Q} \text { and } \frac{\left(\mathcal{H}_{p}^{l, m}\left[\alpha_{1}+2\right] f(z)\right)^{\prime}}{\left(\mathcal{H}_{p}^{l, m}\left[\alpha_{1}+1\right] f(z)\right)^{\prime}} \text { is univalent in } \Delta,
$$


then $f(z) \in \widetilde{\Omega}_{p}\left(\alpha_{1} ; \psi\right)$.

Proof. First of all consider the following identity

$$
z\left(\mathcal{H}_{p}^{l, m}\left[\alpha_{1}\right] f(z)\right)^{\prime}=\alpha_{1} \mathcal{H}_{p}^{l, m}\left[\alpha_{1}+1\right] f(z)-\left(\alpha_{1}+p\right) \mathcal{H}_{p}^{l, m}\left[\alpha_{1}\right] f(z),
$$

which upon differentiation, yields

$$
z\left(\mathcal{H}_{p}^{l, m}\left[\alpha_{1}\right] f(z)\right)^{\prime \prime}=\alpha_{1}\left(\mathcal{H}_{p}^{l, m}\left[\alpha_{1}+1\right] f(z)\right)^{\prime}-\left(\alpha_{1}+p+1\right)\left(\mathcal{H}_{p}^{l, m}\left[\alpha_{1}\right] f(z)\right)^{\prime} .
$$

Now define the function $q(z)$ by

$$
q(z):=\frac{\left(\mathcal{H}_{p}^{l, m}\left[\alpha_{1}+1\right] f(z)\right)^{\prime}}{\left(\mathcal{H}_{p}^{l, m}\left[\alpha_{1}\right] f(z)\right)^{\prime}} .
$$

Then, clearly, $q(z)$ is analytic in $\Delta$.

By logarithmic differentiation of (3.5) with respect to $z$ and using (3.4), we obtain

$$
\frac{\left(\mathcal{H}_{p}^{l, m}\left[\alpha_{1}+2\right] f(z)\right)^{\prime}}{\left(\mathcal{H}_{p}^{l, m}\left[\alpha_{1}+1\right] f(z)\right)^{\prime}}=\frac{1}{\alpha_{1}+1}\left(\alpha_{1} q(z)+1+\frac{z q^{\prime}(z)}{q(z)}\right) .
$$

Since $f(z) \in \Omega_{p}\left(\alpha_{1}+1 ; \chi\right)$, we have from (3.6) that

$$
\alpha_{1} q(z)+\frac{z q^{\prime}(z)}{q(z)} \prec \alpha_{1} \psi(z)+\frac{z \psi^{\prime}(z)}{\psi(z)}
$$

and this can be written as (2.1), by defining

$$
\vartheta(w):=\alpha_{1} w \text { and } \varphi(w):=\frac{1}{w} .
$$

Note that $\varphi(w) \neq 0$ and $\vartheta(w), \varphi(w)$ are analytic in $\mathbb{C} \backslash\{0\}$. Set

$$
\begin{aligned}
Q(z) & :=\frac{z \psi^{\prime}(z)}{\psi(z)} \\
h(z) & :=\vartheta(\psi(z))+Q(z)=\alpha_{1} \psi(z)+\frac{z \psi^{\prime}(z)}{\psi(z)} .
\end{aligned}
$$

In light of the hypothesis of our Theorem 3.1, we see that $Q(z)$ is starlike and

$$
\Re\left\{\frac{z h^{\prime}(z)}{Q(z)}\right\}=\Re\left\{\alpha_{1} \psi(z)+1+\frac{z \psi^{\prime \prime}(z)}{\psi^{\prime}(z)}-\frac{z \psi^{\prime}(z)}{\psi(z)}\right\}>0 .
$$

By an application of Lemma 2.1, we obtain that $q(z) \prec \psi(z)$ or

$$
\frac{\left(\mathcal{H}_{p}^{l, m}\left[\alpha_{1}+1\right] f(z)\right)^{\prime}}{\left(\mathcal{H}_{p}^{l, m}\left[\alpha_{1}\right] f(z)\right)^{\prime}} \prec \psi(z),
$$


which shows that $f(z) \in \Omega_{p}\left(\alpha_{1} ; \psi\right)$.

The second half of the Theorem 3.1 follows by a similar application of Lemma 2.2.

Remark 3.1. The subordination result of Theorem 3.1 also holds if we replace the condition $\Re\left\{\alpha_{1} \psi(z)\right\}>0$ by

$$
\Re\left\{\alpha_{1} \psi(z)+1+\frac{z \psi^{\prime \prime}(z)}{\psi^{\prime}(z)}-\frac{z \psi^{\prime}(z)}{\psi(z)}\right\}>0
$$

and hence we obtain as its special case the following results using (1.3) and (1.4) respectively:

(1) Let $\psi(z)$ be univalent in $\Delta, \psi(0)=1$ and $\psi(z) \neq 0$. Assume that $z \psi^{\prime} / \psi$ is starlike in $\Delta$ and

$$
\Re\left\{a \psi(z)+1+\frac{z \psi^{\prime \prime}(z)}{\psi^{\prime}(z)}-\frac{z \psi^{\prime}(z)}{\psi(z)}\right\}>0 .
$$

Let $\chi(z)$ be defined by

$$
\chi(z):=\frac{1}{a+1}\left[a \psi(z)+1+\frac{z \psi^{\prime}(z)}{\psi(z)}\right] .
$$

If $f(z) \in \Sigma_{p}$ and

$$
\frac{\left[\mathcal{L}_{p}(a+2, c) f(z)\right]^{\prime}}{\left[\mathcal{L}_{p}(a+1, c) f(z)\right]^{\prime}} \prec \chi(z)
$$

then

$$
\frac{\left[\mathcal{L}_{p}(a+1, c) f(z)\right]^{\prime}}{\left[\mathcal{L}_{p}(a, c) f(z)\right]^{\prime}} \prec \psi(z)
$$

and $\psi(z)$ is the best dominant.

(2) Let $\psi(z)$ be univalent in $\Delta, \psi(0)=1$ and $\psi(z) \neq 0$. Assume that $z \psi^{\prime} / \psi$ is starlike in $\Delta$ and

$$
\Re\left\{(n+p) \psi(z)+1+\frac{z \psi^{\prime \prime}(z)}{\psi^{\prime}(z)}-\frac{z \psi^{\prime}(z)}{\psi(z)}\right\}>0 .
$$

Let $\chi(z)$ be defined by

$$
\chi(z):=\frac{1}{n+p+1}\left[(n+p) \psi(z)+1+\frac{z \psi^{\prime}(z)}{\psi(z)}\right] .
$$

If $f(z) \in \Sigma_{p}$ and

$$
\frac{\left[\mathcal{D}^{n+p+1} f(z)\right]^{\prime}}{\left[\mathcal{D}^{n+p} f(z)\right]^{\prime}} \prec \chi(z)
$$

then

$$
\frac{\left[\mathcal{D}^{n+p} f(z)\right]^{\prime}}{\left[\mathcal{D}^{n+p-1} f(z)\right]^{\prime}} \prec \psi(z)
$$

and $\psi(z)$ is the best dominant. 
Using Theorem 3.1, we obtain the following "sandwich result":

Corollary 3.2. Let $\psi_{i}(z) \neq 0(i=1,2)$ be univalent in $\Delta$. Further assume that $z \psi_{i}^{\prime}(z) / \psi_{i}(z)(i=1,2)$ is starlike univalent in $\Delta$ and $\Re\left\{\alpha_{1} \psi_{i}(z)\right\}>0$. If $f(z) \in \Omega_{p}\left(\alpha_{1}+1 ; \chi_{1}, \chi_{2}\right)$ satisfies (3.2), then $f(z) \in \Omega_{p}\left(\alpha_{1} ; \psi_{1}, \psi_{2}\right)$, where

$$
\chi_{i}(z):=\frac{1}{\alpha_{1}+1}\left[\alpha_{1} \psi_{i}(z)+1+\frac{z \psi_{i}^{\prime}(z)}{\psi_{i}(z)}\right] \quad(i=1,2) .
$$

Theorem 3.3. Let $\psi$ be univalent in $\Delta, \psi(0)=1$, and $\lambda$ be a complex number. Assume that $z \psi^{\prime} /\left(\lambda-p-\alpha_{1}+\alpha_{1} \psi\right)$ is starlike in $\Delta$ and $\Re\left\{\lambda-p-\alpha_{1}+\alpha_{1} \psi(z)\right\}>$ 0 . Define the functions $\mathcal{F}$ and $h$ by

$$
\begin{gathered}
\mathcal{F}(z):=\frac{\lambda-p}{z^{\lambda}} \int_{0}^{z} t^{\lambda-1} f(t) d t, \\
h(z):=\psi(z)+\frac{z \psi^{\prime}(z)}{\lambda-p-\alpha_{1}+\alpha_{1} \psi(z)} . \\
\text { If } f(z) \in \Omega_{p}\left(\alpha_{1} ; h\right), \text { then } \mathcal{F} \in \Omega_{p}\left(\alpha_{1} ; \psi\right) \text {. If } f(z) \in \widetilde{\Omega}_{p}\left(\alpha_{1} ; h\right),
\end{gathered}
$$$$
\text { (3.10) } 0 \neq \frac{\mathcal{H}_{p}^{l, m}\left[\alpha_{1}+1\right] \mathcal{F}(z)}{\mathcal{H}_{p}^{l, m}\left[\alpha_{1}\right] \mathcal{F}(z)} \in \mathscr{H}_{1} \cap \mathcal{Q} \text { and } \frac{\left(\mathcal{H}_{p}^{l, m}\left[\alpha_{1}+1\right] f(z)\right)^{\prime}}{\left(\mathcal{H}_{p}^{l, m}\left[\alpha_{1}\right] f(z)\right)^{\prime}} \text { is univalent in } \Delta,
$$

then the function $\mathcal{F} \in \widetilde{\Omega}_{p}\left(\alpha_{1} ; \psi\right)$.

Proof. From the definition of $\mathcal{F}(z)$ and (3.4), we obtain that

$$
\begin{aligned}
(\lambda-p) \mathcal{H}_{p}^{l, m}\left[\alpha_{1}\right] f(z) & =\lambda \mathcal{H}_{p}^{l, m}\left[\alpha_{1}\right] \mathcal{F}(z)+z\left(\mathcal{H}_{p}^{l, m}\left[\alpha_{1}\right] \mathcal{F}(z)\right)^{\prime} \\
& =\alpha_{1} \mathcal{H}_{p}^{l, m}\left[\alpha_{1}+1\right] \mathcal{F}(z)+\left(\lambda-p-\alpha_{1}\right) \mathcal{H}_{p}^{l, m}\left[\alpha_{1}\right] \mathcal{F}(z)
\end{aligned}
$$

Define the function $q(z)$ by

$$
q(z):=\frac{\left(\mathcal{H}_{p}^{l, m}\left[\alpha_{1}+1\right] \mathcal{F}(z)\right)^{\prime}}{\left(\mathcal{H}_{p}^{l, m}\left[\alpha_{1}\right] \mathcal{F}(z)\right)^{\prime}}
$$

Then, clearly, $q(z)$ is analytic in $\Delta$. Using (3.11) and (3.12), we have

$$
(\lambda-p) \frac{\left(\mathcal{H}_{p}^{l, m}\left[\alpha_{1}\right] f(z)\right)^{\prime}}{\left(\mathcal{H}_{p}^{l, m}\left[\alpha_{1}\right] \mathcal{F}(z)\right)^{\prime}}=\lambda-p-\alpha_{1}+\alpha_{1} q(z) .
$$

Upon logarithmic differentiation of (3.13) and using (3.4), and (3.12), we get

$$
\frac{\left(\mathcal{H}_{p}^{l, m}\left[\alpha_{1}+1\right] f(z)\right)^{\prime}}{\left(\mathcal{H}_{p}^{l, m}\left[\alpha_{1}\right] f(z)\right)^{\prime}}=q(z)+\frac{z q^{\prime}(z)}{\lambda-p-\alpha_{1}+\alpha_{1} q(z)} .
$$


Since $f(z) \in \Omega_{p}\left(\alpha_{1} ; h\right)$, we have, from (3.14),

$$
q(z)+\frac{z q^{\prime}(z)}{\lambda-p-\alpha_{1}+\alpha_{1} q(z)} \prec \psi(z)+\frac{z \psi^{\prime}(z)}{\lambda-p-\alpha_{1}+\alpha_{1} \psi(z)}
$$

and this can be written as (2.1), by defining

$$
\vartheta(w):=w \text { and } \varphi(w):=\frac{1}{\lambda-p-\alpha_{1}+\alpha_{1} w} .
$$

The first half of Theorem 3.3 now follows by an application of Lemma 2.1 and the second half follows by a similar application of Lemma 2.2.

Remark 3.2. The subordination result of Theorem 3.3 also holds if we replace the condition $\Re\left\{\lambda-p-\alpha_{1}+\alpha_{1} \psi(z)\right\}>0$ by

$$
\Re\left\{\lambda-p-\alpha_{1}+\alpha_{1} \psi(z)+1+\frac{z \psi^{\prime \prime}(z)}{\psi^{\prime}(z)}-\frac{\alpha_{1} z \psi^{\prime}(z)}{\lambda-p-\alpha_{1}+\alpha_{1} \psi(z)}\right\}>0
$$

and hence we obtain as its special case the following results using (1.3) and (1.4) respectively:

(1) Let $\psi$ be univalent in $\Delta, \psi(0)=1$, and $\lambda$ be a complex number. Assume that $z \psi^{\prime} /(\lambda-p-a+a \psi)$ is starlike in $\Delta$ and

$$
\Re\left\{\lambda-p-a+a \psi(z)+1+\frac{z \psi^{\prime \prime}(z)}{\psi^{\prime}(z)}-\frac{a z \psi^{\prime}(z)}{\lambda-p-a+a \psi(z)}\right\}>0 .
$$

Let $F$ be defined as in (3.9) and $h(z)$ defined by

$$
h(z):=\psi(z)+\frac{z \psi^{\prime}(z)}{\lambda-p-a+a \psi(z)} .
$$

If $f(z) \in \Sigma_{p}$ and

$$
\frac{\left[\mathcal{L}_{p}(a+1, c) f(z)\right]^{\prime}}{\left[\mathcal{L}_{p}(a, c) f(z)\right]^{\prime}} \prec h(z)
$$

then

$$
\frac{\left[\mathcal{L}_{p}(a+1, c) F(z)\right]^{\prime}}{\left[\mathcal{L}_{p}(a, c) F(z)\right]^{\prime}} \prec \psi(z)
$$

and $\psi(z)$ is the best dominant.

(2) Let $\psi$ be univalent in $\Delta, \psi(0)=1$, and $\lambda$ be a complex number. Assume that $z \psi^{\prime} /(\lambda-n+(n+p) \psi)$ is starlike in $\Delta$ and

$$
\Re\left\{\lambda-n+(n+p) \psi(z)+1+\frac{z \psi^{\prime \prime}(z)}{\psi^{\prime}(z)}-\frac{(n+p) z \psi^{\prime}(z)}{\lambda-n+(n+p) \psi(z)}\right\}>0 .
$$


Let $F$ be defined as in (3.9) and $h(z)$ defined by

$$
h(z):=\psi(z)+\frac{z \psi^{\prime}(z)}{\lambda-n+(n+p) \psi(z)} .
$$

If $f(z) \in \Sigma_{p}$ and

$$
\frac{\left[\mathcal{D}^{n+p} f(z)\right]^{\prime}}{\left[\mathcal{D}^{n+p-1} f(z)\right]^{\prime}} \prec h(z)
$$

then

$$
\frac{\left[\mathcal{D}^{n+p} F(z)\right]^{\prime}}{\left[\mathcal{D}^{n+p-1} F(z)\right]^{\prime}} \prec \psi(z)
$$

and $\psi(z)$ is the best dominant.

Using Theorem 3.3, we have the following result:

Corollary 3.4. Let $\psi_{i}$ be univalent in $\Delta(i=1,2)$ and $\lambda$ be a complex number. Assume that $\frac{z \psi_{i}^{\prime}}{\lambda-p-\alpha_{1}+\alpha_{1} \psi_{i}}$ is starlike in $\Delta$ and $\Re\left\{\lambda-p-\alpha_{1}+\alpha_{1} \psi_{i}(z)\right\}>0$ for $i=1,2$. If $f(z) \in \Omega_{p}\left(\alpha_{1} ; h_{1}, h_{2}\right)$ satisfies (3.10), then the function $\mathcal{F}$ defined by (3.9) belongs to $\Omega_{p}\left(\alpha_{1} ; \psi_{1}, \psi_{2}\right)$ where

$$
h_{i}(z):=\psi_{i}(z)+\frac{z \psi_{i}^{\prime}(z)}{\lambda-p-\alpha_{1}+\alpha_{1} \psi_{i}(z)}(i=1,2) .
$$

Theorem 3.5. Let $f(z) \in \Sigma_{p}$ and $\alpha_{1} \neq-1$. Define $\mathcal{F}$ by

$$
\mathcal{F}(z):=\frac{\alpha_{1}}{z^{\alpha_{1}+p}} \int_{0}^{z} t^{\alpha_{1}+p-1} f(t) d t
$$

Then $f(z) \in \Omega_{p}\left(\alpha_{1} ; \varphi\right)$ if and only if $\mathcal{F} \in \Omega\left(\alpha_{1}+1 ; \frac{1+\alpha_{1} \varphi}{1+\alpha_{1}}\right)$. Also $f(z) \in$ $\widetilde{\Omega}_{p}\left(\alpha_{1} ; \varphi\right)$ if and only if $\mathcal{F} \in \widetilde{\Omega}\left(\alpha_{1}+1 ; \frac{1+\alpha_{1} \varphi}{1+\alpha_{1}}\right)$.

Proof. From (3.15), we have

$$
\alpha_{1} f(z)=\left(\alpha_{1}+p\right) \mathcal{F}(z)+z \mathcal{F}^{\prime}(z)
$$

By convoluting (3.16) with $h_{p}\left(\alpha_{1}, \cdots, \alpha_{l} ; \beta_{1}, \cdots, \beta_{m} ; z\right)$ and using the fact that $z(f * g)^{\prime}(z)=f(z) * z g^{\prime}(z)$, we obtain

$$
\alpha_{1} \mathcal{H}_{p}^{l, m}\left[\alpha_{1}\right] f(z)=\left(\alpha_{1}+p\right) \mathcal{H}_{p}^{l, m}\left[\alpha_{1}\right] \mathcal{F}(z)+z\left(\mathcal{H}_{p}^{l, m}\left[\alpha_{1}\right] \mathcal{F}(z)\right)^{\prime}
$$

and by using (3.4), we get

$$
\mathcal{H}_{p}^{l, m}\left[\alpha_{1}\right] f(z)=\mathcal{H}_{p}^{l, m}\left[\alpha_{1}+1\right] \mathcal{F}(z)
$$


and

$$
\begin{aligned}
\alpha_{1} \mathcal{H}_{p}^{l, m}\left[\alpha_{1}+1\right] f(z)= & z\left(\mathcal{H}_{p}^{l, m}\left[\alpha_{1}\right] f(z)\right)^{\prime}+\left(\alpha_{1}+p\right) \mathcal{H}_{p}^{l, m}\left[\alpha_{1}\right] f(z) \\
= & z\left(\mathcal{H}_{p}^{l, m}\left[\alpha_{1}+1\right] \mathcal{F}(z)\right)^{\prime}+\left(\alpha_{1}+p\right) \mathcal{H}_{p}^{l, m}\left[\alpha_{1}+1\right] \mathcal{F}(z) \\
= & \left(\alpha_{1}+1\right) \mathcal{H}_{p}^{l, m}\left[\alpha_{1}+2\right] \mathcal{F}(z)-\left(\alpha_{1}+p+1\right) \mathcal{H}_{p}^{l, m}\left[\alpha_{1}+1\right] \mathcal{F}(z) \\
& \quad+\left(\alpha_{1}+p\right) \mathcal{H}_{p}^{l, m}\left[\alpha_{1}+1\right] \mathcal{F}(z) \\
= & \left(\alpha_{1}+1\right) \mathcal{H}_{p}^{l, m}\left[\alpha_{1}+2\right] \mathcal{F}(z)-\mathcal{H}_{p}^{l, m}\left[\alpha_{1}+1\right] \mathcal{F}(z) .
\end{aligned}
$$

Therefore, from (3.17) and (3.18), we have

$$
\frac{\left(\mathcal{H}_{p}^{l, m}\left[\alpha_{1}+2\right] \mathcal{F}(z)\right)^{\prime}}{\left(\mathcal{H}_{p}^{l, m}\left[\alpha_{1}+1\right] \mathcal{F}(z)\right)^{\prime}}=\frac{1}{\alpha_{1}+1}\left[1+\alpha_{1} \frac{\left(\mathcal{H}_{p}^{l, m}\left[\alpha_{1}+1\right] f(z)\right)^{\prime}}{\left(\mathcal{H}_{p}^{l, m}\left[\alpha_{1}\right] f(z)\right)^{\prime}}\right]
$$

and the desired results follow at once.

Using (1.3), we have the following result:

Example 3.1. Let $f(z) \in \mathcal{A}_{p}$ and $a \neq-1$. Define $F$ as in (3.15). Then

$$
\frac{\left[\mathcal{L}_{p}(a+1, c) f(z)\right]^{\prime}}{\left[\mathcal{L}_{p}(a, c) f(z)\right]^{\prime}} \prec \varphi(z) \text { if and only if } \frac{\left[\mathcal{L}_{p}(a+2, c) F(z)\right]^{\prime}}{\left[\mathcal{L}_{p}(a+1, c) F(z)\right]^{\prime}} \prec \frac{1+a \varphi}{1+a} \text {. }
$$

Also

$$
\varphi(z) \prec \frac{\left[\mathcal{L}_{p}(a+1, c) f(z)\right]^{\prime}}{\left[\mathcal{L}_{p}(a, c) f(z)\right]^{\prime}} \text { if and only if } \frac{1+a \varphi}{1+a} \prec \frac{\left[\mathcal{L}_{p}(a+2, c) F(z)\right]^{\prime}}{\left[\mathcal{L}_{p}(a+1, c) F(z)\right]^{\prime}} .
$$

Using Theorem 3.5, we have

Corollary 3.6. Let $f(z) \in \Sigma_{p}$ and $\alpha_{1} \neq-1$. Then $f(z) \in \Omega_{p}\left(\alpha_{1} ; \varphi_{1}, \varphi_{2}\right)$ if and only if $\mathcal{F}$ given by (3.15) is in $\Omega\left(\alpha_{1}+1 ; \frac{1+\alpha_{1} \varphi_{1}}{1+\alpha_{1}}, \frac{1+\alpha_{1} \varphi_{2}}{1+\alpha_{1}}\right)$.

4. The classes $\mathcal{M}_{p}(n, \lambda ; \varphi)$ and $\widetilde{\mathcal{M}}_{p}(n, \lambda ; \varphi)$

By making use of Lemma 2.1, we prove the following result:

Theorem 4.1. Let $\psi(z)$ be univalent in $\Delta, \psi(0)=1, \Re \psi(z)>0$ and $z \psi^{\prime} / \psi$ be starlike in $\Delta$. Let $\chi(z)$ be defined by

$$
\chi(z):=\frac{1}{\lambda-p}\left[(\lambda-p) \psi(z)+\frac{z \psi^{\prime}(z)}{\psi(z)}\right] .
$$

If $f(z) \in \mathcal{M}_{p}(n+1, \lambda ; \chi)$, then $f(z) \in \mathcal{M}_{p}(n, \lambda ; \psi)$. If $f(z) \in \widetilde{\mathcal{M}}_{p}(n+1, \lambda ; \chi)$,

(4.1) $0 \neq \frac{\left(\mathcal{I}_{p}(n+1, \lambda) f(z)\right)^{\prime}}{\left(\mathcal{I}_{p}(n, \lambda) f(z)\right)^{\prime}} \in \mathscr{H}_{1} \cap \mathcal{Q}$ and $\frac{\left(\mathcal{I}_{p}(n+2, \lambda) f(z)\right)^{\prime}}{\left(\mathcal{I}_{p}(n+1, \lambda) f(z)\right)^{\prime}}$ is univalent in $\Delta$, 
then $f(z) \in \widetilde{\mathcal{M}}_{p}(n, \lambda ; \psi)$.

Proof. First of all consider the following identity

$$
(\lambda-p) \mathcal{I}_{p}(n+1, \lambda) f(z)=z\left[\mathcal{I}_{p}(n, \lambda) f(z)\right]^{\prime}+\lambda \mathcal{I}_{p}(n, \lambda) f(z),
$$

which yields up on differentiation

$$
z\left[\mathcal{I}_{p}(n, \lambda) f(z)\right]^{\prime \prime}=(\lambda-p)\left[\mathcal{I}_{p}(n+1, \lambda) f(z)\right]^{\prime}-(\lambda+1)\left[\mathcal{I}_{p}(n, \lambda) f(z)\right]^{\prime} .
$$

Now define the function $q(z)$ by

$$
q(z):=\frac{\left(\mathcal{I}_{p}(n+1, \lambda) f(z)\right)^{\prime}}{\left(\mathcal{I}_{p}(n, \lambda) f(z)\right)^{\prime}} .
$$

Then, clearly, $q(z)$ is analytic in $\Delta$. By logarithmic differentiation of (4.4) and using (4.3), we obtain

$$
\frac{\left(\mathcal{I}_{p}(n+2, \lambda) f(z)\right)^{\prime}}{\left(\mathcal{I}_{p}(n+1, \lambda) f(z)\right)^{\prime}}=\frac{1}{\lambda-p}\left((\lambda-p) q(z)+\frac{z q^{\prime}(z)}{q(z)}\right) .
$$

Since $f(z) \in \mathcal{M}_{p}(n+1, \lambda ; \chi)$ and in view of (4.5), we have

$$
(\lambda-p) q(z)+\frac{z q^{\prime}(z)}{q(z)} \prec(\lambda-p) \psi(z)+\frac{z \psi^{\prime}(z)}{\psi(z)} .
$$

The first result follows by an application of Lemma 2.1. Similarly the second result follows from Lemma 2.2.

Remark 4.1. The subordination result of Theorem 4.1 also holds if we replace the condition $\Re \psi(z)>0$ by

$$
\Re\left\{(\lambda-p) \psi(z)+1+\frac{z \psi^{\prime \prime}(z)}{\psi^{\prime}(z)}-\frac{z \psi^{\prime}(z)}{\psi(z)}\right\}>0 .
$$

Using Theorem 4.1, we obtain the following "sandwich result":

Corollary 4.2. Let $\psi_{i}(z)$ be univalent in $\Delta, \Re \psi_{i}(z)>0$ and $z \psi_{i}^{\prime}(z) / \psi_{i}(z)$ be starlike univalent in $\Delta$ for $i=1,2$. Define

$$
\chi_{i}(z):=\frac{1}{\lambda-p}\left[(\lambda-p) \psi_{i}(z)+\frac{z \psi_{i}^{\prime}(z)}{\psi_{i}(z)}\right] \quad(i=1,2) .
$$

If $f(z) \in \mathcal{M}_{p}\left(n+1, \lambda ; \chi_{1}, \chi_{2}\right)$ satisfies (4.1), then $f(z) \in \mathcal{M}_{p}\left(n, \lambda ; \psi_{1}, \psi_{2}\right)$.

Theorem 4.3. Let $\psi$ be univalent in $\Delta, \psi(0)=1, \delta$ be a complex number, $z \psi^{\prime} /(\delta-$ 
$\lambda+(\lambda-p) \psi)$ be starlike in $\Delta$ and $\Re\{\delta-\lambda+(\lambda-p) \psi(z)\}>0$. Define the function $\mathcal{F}$ by

$$
\begin{aligned}
\mathcal{F}(z) & :=\frac{\delta-p}{z^{\delta}} \int_{0}^{z} t^{\delta-1} f(t) d t, \\
h(z) & :=\psi(z)+\frac{z \psi^{\prime}(z)}{\delta-\lambda+(\lambda-p) \psi(z)} .
\end{aligned}
$$

If $f(z) \in \mathcal{M}_{p}(n, \lambda ; h)$, then $\mathcal{F} \in \mathcal{M}_{p}(n, \lambda ; \psi)$. If $f(z) \in \widetilde{\mathcal{M}}_{p}(n, \lambda ; h)$,

$$
0 \neq \frac{\left(\mathcal{I}_{p}(n+1, \lambda) \mathcal{F}(z)\right)^{\prime}}{\left(\mathcal{I}_{p}(n, \lambda) \mathcal{F}(z)\right)^{\prime}}(z) \in \mathscr{H}_{1} \cap \mathcal{Q} \text { and } \frac{\left(\mathcal{I}_{p}(n+1, \lambda) f(z)\right)^{\prime}}{\left(\mathcal{I}_{p}(n, \lambda) f(z)\right)^{\prime}} \text { is univalent in } \Delta,
$$

then $\mathcal{F} \in \widetilde{\mathcal{M}}_{p}(n, \lambda ; \psi)$.

Proof. From the definition of $\mathcal{F}(z)$ and

$$
z\left(\mathcal{I}_{p}(n, \lambda) \mathcal{F}(z)\right)^{\prime}=(\lambda-p) \mathcal{I}_{p}(n+1, \lambda) \mathcal{F}(z)-\lambda \mathcal{I}_{p}(n, \lambda) \mathcal{F}(z),
$$

we have

$$
\begin{aligned}
(\delta-p) \mathcal{I}_{p}(n, \lambda) f(z) & =\delta \mathcal{I}_{p}(n, \lambda) \mathcal{F}(z)+z\left(\mathcal{I}_{p}(n, \lambda) \mathcal{F}(z)\right)^{\prime} \\
& =(\lambda-p) \mathcal{I}_{p}(n+1, \lambda) \mathcal{F}(z)+(\delta-\lambda) \mathcal{I}_{p}(n, \lambda) \mathcal{F}(z) .
\end{aligned}
$$

Define the function $q(z)$ by

$$
q(z):=\frac{\left(\mathcal{I}_{p}(n+1, \lambda) \mathcal{F}(z)\right)^{\prime}}{\left(\mathcal{I}_{p}(n, \lambda) \mathcal{F}(z)\right)^{\prime}}
$$

Then, clearly, $q(z)$ is analytic in $\Delta$. Using (4.9) and (4.10), we have

$$
(\delta-p) \frac{\left(\mathcal{I}_{p}(n, \lambda) f(z)\right)^{\prime}}{\left(\mathcal{I}_{p}(n, \lambda) \mathcal{F}(z)\right)^{\prime}}=\delta-\lambda+(\lambda-p) q(z) .
$$

Upon logarithmic differentiation of (4.11) and using (4.3), (4.8) and (4.10), we get

$$
\frac{\left(\mathcal{I}_{p}(n+1, \lambda) f(z)\right)^{\prime}}{\left(\mathcal{I}_{p}(n, \lambda) f(z)\right)^{\prime}}=q(z)+\frac{z q^{\prime}(z)}{\delta-\lambda+(\lambda-p) q(z)} .
$$

For $f(z) \in \mathcal{M}_{p}(n, \lambda, h)$, we have from (4.11),

$$
q(z)+\frac{z q^{\prime}(z)}{\delta-\lambda+(\lambda-p) q(z)} \prec \psi(z)+\frac{z \psi^{\prime}(z)}{\delta-\lambda+(\lambda-p) \psi(z)} .
$$

The first part of our result now follows by an application of Lemma 2.1. Similarly the second part follows from Lemma 2.2 . 
Remark 4.2. The subordination result of Theorem 4.3 also holds if we replace the condition $\Re\{\delta-\lambda+(\lambda-p) \psi(z)\}>0$ by

$$
\Re\left\{\delta-\lambda+(\lambda-p) \psi(z)+1+\frac{z \psi^{\prime \prime}(z)}{\psi^{\prime}(z)}-\frac{(\lambda-p) z \psi^{\prime}(z)}{\delta-\lambda+(\lambda-p) \psi(z)}\right\}>0 .
$$

Using Theorem 4.3, we have the following result:

Corollary 4.4. Let $\psi_{i}$ be univalent in $\Delta$ and $\delta$ be a complex number. Assume that $z \psi_{i}^{\prime} /\left(\delta-\lambda+(\lambda-p) \psi_{i}\right)$ is starlike in $\Delta$ and $\Re\left\{\delta-\lambda+(\lambda-p) \psi_{i}(z)\right\}>0$ for $i=1,2$. Define the functions $h_{i}$ by

$$
h_{i}(z):=\psi_{i}(z)+\frac{z \psi_{i}^{\prime}(z)}{\delta-\lambda+(\lambda-p) \psi_{i}(z)}(i=1,2) .
$$

If $f(z) \in \mathcal{M}_{p}\left(n, \lambda ; h_{1}, h_{2}\right)$, then $\mathcal{F}$ defined by (4.6) belongs to $\mathcal{M}_{p}\left(n, \lambda ; \psi_{1}, \psi_{2}\right)$.

Theorem 4.5. Let $f(z) \in \Sigma_{p}$. Then $f(z) \in \mathcal{M}_{p}(n, \lambda ; \varphi)$ if and only if

$$
\mathcal{F}(z):=\frac{\lambda-p}{z^{\lambda}} \int_{0}^{z} t^{\lambda-1} f(t) d t \in \mathcal{M}_{p}(n+1, \lambda ; \varphi) .
$$

Also $f(z) \in \widetilde{\mathcal{M}}_{p}(n, \lambda ; \varphi)$ if and only if $\mathcal{F} \in \widetilde{\mathcal{M}}_{p}(n+1, \lambda ; \varphi)$.

Proof. From (4.13), we have

$$
(\lambda-p) f(z)=\lambda \mathcal{F}(z)+z \mathcal{F}^{\prime}(z) .
$$

By convoluting (4.14) with

$$
\phi_{p}(k, \lambda ; z):=\frac{1}{z^{p}}+\sum_{k=1-p}^{\infty}\left(\frac{k+\lambda}{\lambda-p}\right)^{r} z^{k}
$$

and using the fact that $z(f * g)^{\prime}(z)=f(z) * z g^{\prime}(z)$, we obtain

$$
(\lambda-p) \mathcal{I}_{p}(n, \lambda) f(z)=\lambda \mathcal{I}_{p}(n, \lambda) \mathcal{F}(z)+z\left(\mathcal{I}_{p}(n, \lambda) \mathcal{F}(z)\right)^{\prime}
$$

and by using (4.3), we get

$$
\mathcal{I}_{p}(n, \lambda) f(z)=\mathcal{I}_{p}(n+1, \lambda) \mathcal{F}(z)
$$

and

$$
\begin{aligned}
(\lambda-p) \mathcal{I}_{p}(n+1, \lambda) f(z) & =z\left(\mathcal{I}_{p}(n, \lambda) f(z)\right)^{\prime}+\lambda \mathcal{I}_{p}(n, \lambda) f(z) \\
& =z\left(\mathcal{I}_{p}(n+1, \lambda) \mathcal{F}(z)\right)^{\prime}+\lambda \mathcal{I}_{p}(n+1, \lambda) \mathcal{F}(z) \\
& =(\lambda-p) \mathcal{I}_{p}(n+2, \lambda) \mathcal{F}(z) .
\end{aligned}
$$


Therefore, from (4.15) and (4.16), we have

$$
\frac{\left(\mathcal{I}_{p}(n+2, \lambda) \mathcal{F}(z)\right)^{\prime}}{\left(\mathcal{I}_{p}(n+1, \lambda) \mathcal{F}(z)\right)^{\prime}}=\frac{\left(\mathcal{I}_{p}(n+1, \lambda) f(z)\right)^{\prime}}{\left(\mathcal{I}_{p}(n, \lambda) f(z)\right)^{\prime}},
$$

and the desired result follows at once.

Using Theorem 4.5, we have

Corollary 4.6. Let $f(z) \in \Sigma_{p}$. Then $f(z) \in \mathcal{M}_{p}\left(n, \lambda ; \varphi_{1}, \varphi_{2}\right)$ if and only if $\mathcal{F} \in \mathcal{M}_{p}\left(n+1, \lambda ; \varphi_{1}, \varphi_{2}\right)$.

\section{References}

[1] M. K. Aouf and H. M. Hossen, New criteria for meromorphic p-valent starlike functions, Tsukuba J. Math., 17(2)(1993), 481-486.

[2] T. Bulboacă, A class of superordination-preserving integral operators, Indag. Math. (N. S.), 13(3)(2002), 301-311.

[3] T. Bulboacă, Classes of first-order differential superordinations, Demonstratio Math., 35(2)(2002), 287-292.

[4] N. E. Cho and S. H. Lee, Certain subclasses of meromorphic functions defined by subordination. II, Kyungpook Math. J., 36(2)(1996), 283-291.

[5] M. R. Ganigi and B. A. Uralegaddi, New criteria for meromorphic univalent functions, Bull. Math. Soc. Sci. Math. R. S. Roumanie (N. S.), 33(81)(1)(1989), 9-13.

[6] J. -L. Liu, A linear operator and its applications on meromorphic p-valent functions, Bull. Inst. Math. Acad. Sinica, 31(1)(2003), 23-32.

[7] J. -L. Liu and H. M. Srivastava, Classes of meromorphically multivalent functions associated with the generalized hypergeometric function, Math. Comput. Modelling, 39(1)(2004), 21-34.

[8] S. S. Miller and P. T. Mocanu, Differential subordinations, Monographs and Textbooks in Pure and Applied Mathematics, vol. 225, Marcel Dekker Inc., New York, 2000.

[9] S. S. Miller and P. T. Mocanu, Subordinants of differential superordinations, Complex Var. Theory Appl., 48(10)(2003), 815-826.

[10] J. Patel and P. Sahoo, On certain subclasses of meromorphically p-valent functions, Bull. Calcutta Math. Soc., 93(6)(2001), 455-464.

[11] V. Ravichandran, S. Sivaprasad Kumar, and K. G. Subramanian, Convolution conditions for spirallikeness and convex spirallikeness of certain meromorphic p-valent functions, JIPAM. J. Inequal. Pure Appl. Math., 5(1)(2004), Article 11, 7 pp. (electronic).

[12] B. A. Uralegaddi and C. Somanatha, New criteria for meromorphic starlike univalent functions, Bull. Austral. Math. Soc., 43(1)(1991), 137-140. 
[13] D. Yang, On new subclasses of meromorphic p-valent functions, J. Math. Res. Exposition, 15(1)(1995), 7-13.

[14] D. Yang, On a class of meromorphic starlike multivalent functions, Bull. Inst. Math. Acad. Sinica, 24(2)(1996), 151-157. 